\title{
Super-roughening as a disorder-dominated flat phase
}

\author{
S. Ares ${ }^{1}$, A. SÁnchez ${ }^{1}$ and A. R. Bishop ${ }^{2}$ \\ 1 Grupo Interdisciplinar de Sistemas Complejos $\left(\right.$ GISC) $\left({ }^{*}\right)$ and \\ Departamento de Matemáticas, Universidad Carlos III de Madrid \\ Avenida de la Universidad 30, 28911 Leganés, Madrid, Spain \\ 2 Theoretical Division and Center for Nonlinear Studies, MS B258 \\ Los Alamos National Laboratory, Los Alamos, NM 87545, USA
}

PACS. 68.35.Ct - Interface structure and roughness.

PACS. 68.35.Rh - Phase transitions and critical phenomena.

PACS. 05.40.-a - Fluctuation phenomena, random processes, noise, and Brownian motion.

\begin{abstract}
We study the phenomenon of super-roughening found on surfaces growing on disordered substrates. We consider a one-dimensional version of the problem for which the pure, ordered model exhibits a roughening phase transition. Extensive numerical simulations combined with analytical approximations indicate that super-roughening is a regime of asymptotically flat surfaces with non-trivial, rough short-scale features arising from the competition between surface tension and disorder. Based on this evidence and on previous simulations of the two-dimensional random sine-Gordon model (Sánchez et al., Phys. Rev. E, 62 (2000) 3219), we argue that this scenario is general and explains equally well the hitherto poorly understood two-dimensional case.
\end{abstract}

Understanding phase transitions in systems with quenched disorder is a challenging issue, not only in physics but also in fields ranging from combinatorics to financial market modeling. This is the reason why much effort has been devoted in the last few years to this subject. However, the progress achieved so far is modest, and clear-cut, definitive results are rare [1]. In view of this, steps towards providing such results are needed and will be very helpful for many ongoing investigations. One important instance where the role of disorder remains unclear is that of roughening transitions of crystal surfaces [2]: At high temperatures, crystal surfaces are rough (the surface width $w$ increases with the system size $L$ ), whereas at low temperatures they become flat ( $w$ is a constant depending only on temperature). When crystal planes are grown on a disordered substrate, renormalization group calculations for the two-dimensional (2D) random sine-Gordon model (RsGM, see below) predicted that the conventional roughening transition was replaced by super-roughening [3], a new phase transition from the high-temperature rough phase $(w \sim \sqrt{\ln L})$ to a low-temperature rougher phase $(w \sim \ln L)$. Subsequent work produced a "myriad of predictions" [4] in a few years, which gave rise to a controversy about the nature of the super-rough phase (see [5] for a review). Based mainly on large-scale numerical simulations or exact optimization results [6-14], some degree of consensus was reached that super-roughening indeed took place and that $w \sim \ln L$

$(*)$ http://gisc.uc3m.es 
was observed. However, recent numerical evidence from simulations by our group on the 2D RsGM [15] has cast doubts on the generality of that behavior as the parameters of the model change, suggesting that the low-temperature phase of the model could even be flat.

With the expectation that the problem would be more amenable both analytically and numerically, in this letter we study the same problem in a one-dimensional (1D) setting. To mimic as closely as possible the 2D situation, we need to find a $1 \mathrm{D}$ model whose non-random version exhibits a true roughening transition. The 1D sine-Gordon model does not fulfill this condition (see [16] for a rigorous proof; see also [17] for additional numerical results) and therefore we must modify it. To this end, we introduce and study for the first time a new model, rooted in the work of Burkhardt [18], who proved that the Hamiltonian given by (periodic boundary conditions on a lattice with $N$ sites are used) $\mathcal{H}=\sum_{i=1}^{N}\left\{J\left|h_{i+1}-h_{i}\right|+U\left(h_{i}\right)\right\}$, with $U(x)$ being a square well potential, $U(x)=-U_{0}$ for $0<x<R, U(x)=0$ otherwise, and with the heights $h_{i}$ restricted to be non-negative (impenetrable substrate) has a roughening transition (see [19] for analogous discrete models). We stress that this is a true thermodynamic phase transition, which is perfectly possible in 1D (see [20] for a thorough discussion of $1 \mathrm{D}$ phase transitions). Building on these results, we propose the following Hamiltonian as our basic model (hereafter called Burkhardt-RsGM or B-RsGM):

$$
\mathcal{H}=\sum_{i=1}^{N}\left\{\frac{1}{2}\left(h_{i+1}-h_{i}\right)^{2}+\left[V\left(h_{i}-h_{i}^{(0)}\right)\right]\right\}
$$

where $V(x) \equiv V_{0}(1-\cos x)+U(x), h_{i}^{(0)}$ are uncorrelated random variables uniformly distributed in the range $\left[0, h_{\max }\right]$, and the impenetrable substrate restriction now reading $h_{i}^{(0)} \leq$ $h_{i} \leq \infty$. We note that the original proposal of Burkhardt had a surface tension term given by $\left|h_{i+1}-h_{i}\right|$. Instead, model (1) follows the spirit of the RsGM, including surface tension as the square of a discrete gradient term [21] and a periodic potential favoring multiples of $2 \pi$ for the heights $h_{i}$, mimicking the growth of crystal layers. The $1 \mathrm{D}$ RsGM is recovered by setting $U_{0}=0$ and allowing for negative heights.

We have not been able to solve the statistical mechanics of the ordered B-RsGM (i.e., $\left.h_{\max }=0\right)$ exactly. Therefore, we have resorted to numerical simulations to check whether the roughening transition in the original Burkhardt model carries over to our modified version. To this end, we have used parallel tempering Monte Carlo [22,23]. Representative configurations at a given temperature are generated with a heat bath algorithm [24], full details of which can be found in [17]. The parallel tempering algorithm then considers simultaneous copies of the system at different temperatures, allowing exchange of configurations between them. This is particularly efficient for low-temperature configurations, which are most susceptible to being trapped in metastable regions, particularly in the disordered case.

The results of our simulations of the B-RsGM without disorder are summarized in fig. 1 for the specific choice $V_{0}=1, U_{0}=2, R=2 \pi$ (to have the same length as the periodicity of the cosine term), and $h_{\max }=2 \pi$ (as in the standard 2D RsGM); other values give qualitatively the same results. Our systems have sizes in the range between 500 and 2000 sites. As can be seen from the plot, the specific heat presents a clear jump at a temperature $T_{\mathrm{R}}^{\text {ord }}$ around 10.3 in our units (the peak at lower temperatures corresponds to a well-known Schottky anomaly, see, e.g., [15] and references therein). At the same temperature, the width or roughness, defined as $w^{2} \equiv\left\langle\left[h_{i}-\left\langle h_{i}\right\rangle\right]^{2}\right\rangle$ with $\langle\cdots\rangle$ denoting thermal averages, jumps from values close to zero to large, system-size-dependent values, indicating the onset of the rough phase. This is further confirmed by the height difference correlation function, $C(r) \equiv\left\langle\sum_{i}\left[h_{i}-h_{i+r}\right]^{2}\right\rangle / N$, as shown in the inset of the left panel of fig. 2. Note that the correlation function is scaled 


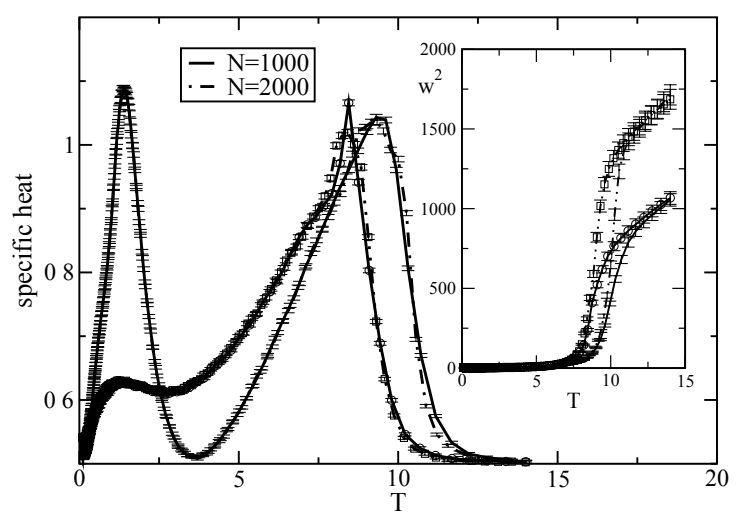

Fig. 1 - Specific heat vs. temperature for the B-RsGM for two system sizes, as indicated. Inset: roughness vs. temperature. Lines with and without symbols correspond to the disordered and ordered case, respectively. Error bars correspond to the thermal average.

by the temperature, aiming to identify the onset of the rough phase as the point at which the curves for different temperatures collapse and become simply proportional to $r$ (the position along the chain). From both plots we locate the transition at $T_{\mathrm{R}}^{\text {ord }}=10.3$; actually, there is a gap in the plot of $C(r) / T$ at $T_{\mathrm{R}}^{\text {ord }}$, and for temperatures slightly above this value the curves go discontinuously to the collapsed, high-temperature one. In this rough phase, the model behaves effectively as though $U_{0}=V_{0}=0$ (in other words, as the Gaussian model). All these features are exactly what we expected from the theoretical results of the original Burkhardt model [18], and make us confident that the B-RsGM presents a roughening phase transition in the absence of disorder, very much like the 2D RsGM. Further confirmation of the existence of this phase transition and of $T_{\mathrm{R}}^{\mathrm{ord}}$ can be obtained by analytical calculations based on a continuum approach leading to a pseudo-Schrödinger equation (following [25]), as well as from numerical evaluation of an exact transfer matrix calculation [26].
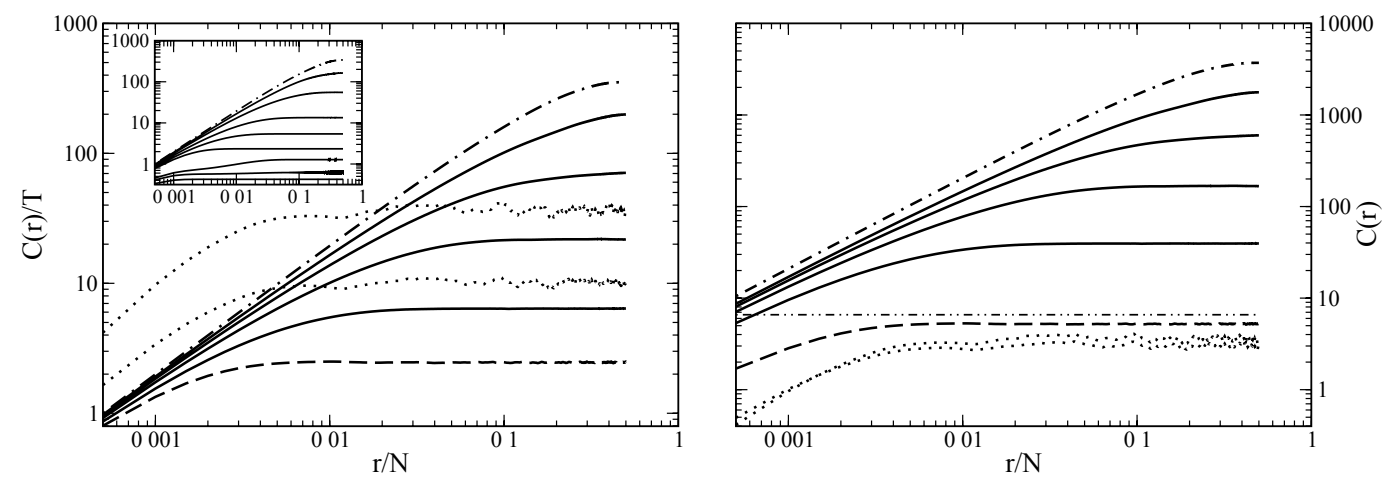

Fig. 2 - Height difference correlation function scaled by the temperature $T$ (left panel) and without scaling (right panel). Temperatures are $T=10.50$ (dot-dashed line), $T=8.84,8.41,7.70,6.17$ (solid lines), $T=2.13$ (dashed line), and $T=0.301,0.0986$ (dotted lines). Inset: scaled height difference correlation function without disorder. Temperatures are $T=14.0$ (dot-dashed line) and $T=10.0$, $9.34,8.18,6.90,3.99,0.995,0.774$, and 0.0981 (solid lines). The double-dot-dashed horizontal line indicates $C(r)$ for a surface exactly locked to the disorder, $C^{(0)}(r)$ (see the text). 


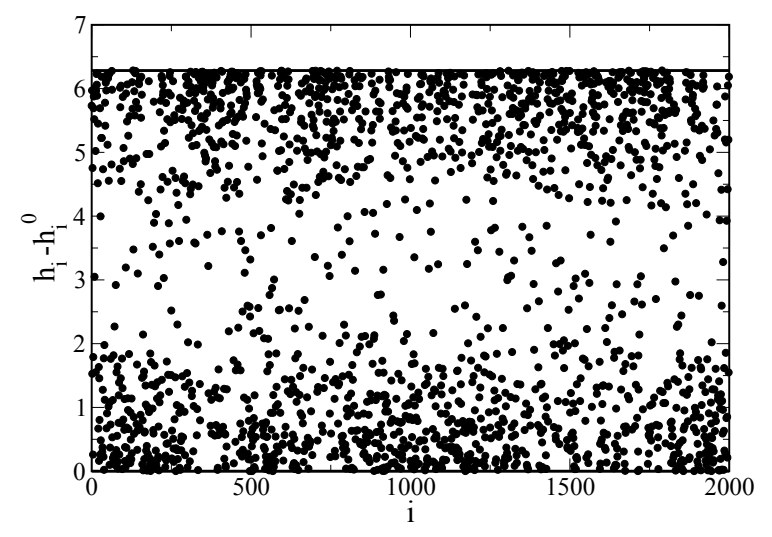

Fig. 3 - Heights above the substrate, $h_{i}-h_{i}^{(0)}$, vs. position along the chain. Temperature is $T=0.0986$, system size $N=2000$.

Having established the existence of a roughening transition in the ordered B-RsGM, we can now proceed to discuss how the disorder changes this transition. Below, we present results of single realization simulations; we have checked that these are typical by running several simulations with different quenched disorder for each case. Figure 1 and the left panel of fig. 2 show that the roughening transition of the ordered model is basically unchanged (although the Schottky peak is much less pronounced, due to the distortion of kinks by the disorder); the value of $T_{\mathrm{R}}$ does change, however, and it is now located at $T_{\mathrm{R}}=9.3$. Furthermore, as the temperature is lowered and reaches $T_{\mathrm{D}}=2.1, C(r) / T$ begins to increase while maintaining a finite correlation length (that implies a flat phase with roughness independent of the system size for sizes larger than the correlation length). This increase arises from the fact that, as seen in the right panel in fig. 2 with the temperature scaling removed, $C(r)$ approaches a constant function, independent of $T$, as $T$ goes to zero, and hence scaling by $T$ leads to an increase of the function. The $1 \mathrm{D}$ character of our model allows us to study large systems and ensure that $C(r)$ indeed has finite range. The value of $T$ at which this change of behavior takes place can be related to the heights taking values close to the disorder ones: In the absence of surface tension (the height differences term), the minimum of $\mathcal{H}$ would be $h_{i}=h_{i}^{(0)}$ for all sites $i$; the correlation function for such a configuration can be immediately found analytically, leading to a constant value $C^{(0)}(r)=2 w^{2}$ independently of the spatial dimension, with $w^{2} \equiv\left\langle\left(h_{i}^{(0)}\right)^{2}\right\rangle-\left\langle h_{i}^{(0)}\right\rangle^{2}=h_{\max }^{2} / 12 \equiv C_{\mathrm{D}}$ being the squared roughness. As can be seen from fig. 2, the scaled $C(r)$ begins to increase precisely when its asymptotic value coincides with $C^{(0)}(r)$. This strongly suggests that at $T_{\mathrm{D}}$ and below, the surface approaches the values of the disorder, while smoothed at short scales by surface tension. Figure 3 confirms this expectation by showing that for a majority of sites $h_{i}$ is close to $h_{i}^{(0)}$ or to $h_{i}^{(0)}+2 \pi\left(h_{i}\right.$ is contained within that range at low temperatures because of Burkhardt's square well). It is clear that the effect of surface tension smooths the surface and leads to a global value for the roughness which is lower than that corresponding to the disorder values. In fact, by analyzing the overdamped equations of motion for $\mathcal{H}$ linearized around the substrate, it is straightforward to show that $C(r)$ goes asymptotically to a nonzero constant $C_{0}$ at low temperatures, in agreement with these numerical simulations (further details will be given elsewhere [27]). Interestingly, at temperatures as low as that in fig. 3, the roughness is slightly larger than at higher temperatures, as seen in fig. 4. This surprising result arises, in our view, from this competition between surface tension and disorder, which for almost zero temperatures seems to favor the latter. 


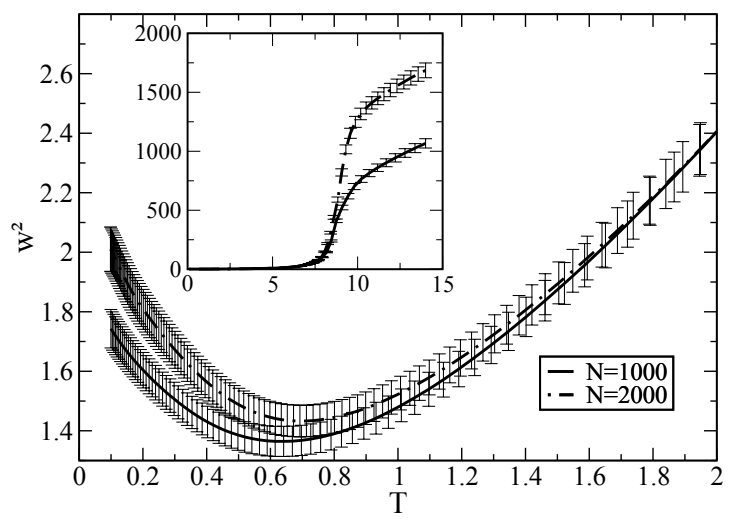

Fig. 4 - Roughness as a function of temperature for the disordered model. Inset: same, but for a larger range of temperatures.

From the evidence summarized above, we conclude that our 1D model shows no signatures of super-roughening. Instead, the picture that emerges from this work is that of a usual roughening transition, with temperature $T_{\mathrm{R}}$ lower than the ordered case, followed at temperatures of the order of or below $T_{\mathrm{D}}<T_{\mathrm{R}}$ by a crossover to a disorder-dominated but otherwise flat phase. In this last regime, the surface is located close to the values imposed by the disorder (modulo $2 \pi$ ). This behavior leads to a height-difference correlation function, $C(r)$, that is asymptotically flat, but increasing at short distances due to surface tension, up to a correlation length of the order of 10-20 lattice units. At larger scales, $C(r)$ is well described analytically by assuming that the surface height takes values close to the disorder. Furthermore, as a consequence of the competition between disorder and surface tension, the width exhibits a non-trivial behavior with a minimum at a low but non-zero temperature.

We believe that our conclusions are relevant in a much broader context than the 1D model we have described. As our model has a true roughening phase transition, it is reasonable to consider it in the related context of the $2 \mathrm{D}$ problem. In the following, we will discuss the results on our 1D model we have presented above together with our previous, extensive numerical work on the 2D RsGM reported in [15]. We encourage the reader to consult [15] as the plots, results and conclusions in that paper, which would be too lengthy to repeat here, clearly suggest that our interpretation is consistent with the available facts about the 2D RsGM. Indeed, we believe that the abundant but often contradictory evidence available regarding the $2 \mathrm{D}$ RsGM can be understood within the scheme proposed here. The key point is that those 2D RsGM simulations [15] are perfectly consistent with our scenario: They show asymptotically flat correlation functions (with constant value approximately given by $C_{0}$ ) and non-monotonic roughness, especially for strong potentials (large values of $V_{0}$; cf. fig. 12 in [15]). It is true, however, that for values of $V_{0}$ of the order of unity a squared logarithm behavior is found for $C(r)$. In the interpretation stemming from the results reported above, this could be due to two factors: First, for such values of $V_{0}, T_{\mathrm{R}} \gtrsim T_{\mathrm{D}}$, and hence the flat phase of the ordered model is not observed; instead, in the corresponding $C(r) / T$ plots in [15] all that appears is that $C(r) / T$ increases with decreasing $T$ because, as discussed above, $C(r)$ has become independent of $T$ and governed by the disorder, with the appearance of super-roughening. Second, in 2D the surface tension influence is much larger than in $1 \mathrm{D}$ because of the increased number of neighbors; as a consequence, the crossover in $C(r)$ from $C(r=0)=0$ to $C(r \rightarrow \infty)=C_{\mathrm{D}}$ is much more pronounced and occurs on a longer spatial 
scale. This is in agreement with the observation in [12] that at $T=0 C(r)$ also exhibits a finite correlation length. Our interpretation is also consistent with the result that changes in $V_{0}$ (also reported in $[4,6,13])$ or in the interval of the disorder $h_{\max }$ lead to the disappearance of the super-rough phase [15], replaced by a phenomenology very close to the one we are proposing. Importantly, we have verified with the results in [15] that the criterion based on comparison with the tensionless $C^{(0)}(r)$ discussed above, works equally well to determine the entrance to the disorder-dominated regime in $2 \mathrm{D}$. We have checked that this is the case by applying the criterion to our results in [15] (cf. specially figs. 3(b) and 7, in complete agreement with the $1 \mathrm{D}$ results). Furthermore, the simulations in [15] yielded non-monotonic behaviors for the total roughness, as depicted in fig. 4 for the 1D model. Finally, another piece of evidence in favor of our proposal is that sudden quenches of surfaces in the 2D RsGM lead to long-lived states with $C(r) \sim \ln ^{3} r$ and other anomalous behaviors [28]. This may be related to the fact that in a quench only very short-scale rearrangements of the surface are possible, leading to a shorter correlation length and a shorter interval for $C(r)$ to rise from 0 to $C_{\mathrm{D}}$.

Based on the above considerations, we believe that our central proposal, namely that superroughening is an effective short-scale phenomenon arising from the existence of a roughening transition and a crossover to a disorder-dominated regime, is very appealing. It would naturally explain the failures of the different theoretical approaches, the predictions of lack of universality, and the discrepancies among the simulations [5]. Thus, obtaining different numerical results is generally the case when studying crossover phenomena, as these are very dependent on the details of the model and even of the simulations. On the other hand, the $\ln ^{2} r$ behavior for $C(r)$ can indeed be found if the scales analyzed are restricted to a small range, such as those reported in [6-11], obtained on systems of at most $200 \times 200$ sites. Our own simulations on systems of sizes up to $256 \times 256$ [15] $(1024 \times 1024$ at zero temperature [12]) confirm partly that result but show indications of a crossover to a flat behavior at larger time scales. Our proposal is an alternative explanation to the currently available results on the phenomenon of super-roughening, but cannot be considered as the definitive answer to this problem without further very large-scale simulations on the $2 \mathrm{D}$ problem. One reason for this caveat is that the nature of the phase transition in our $1 \mathrm{D}$ model is not exactly that of the 2D model, and the models themselves are not identical. We have considered this difficulty, and concluded that the many analogies between the numerical results reported here and those in [15] lead us to suggest on solid grounds that the mechanism underlying this common phenomenology is basically the same. Caution must also be taken as it could be argued that our results carry over only to the 2D B-RsGM and not to the 2D RsGM. In this respect, as the 2D RsGM has a roughening transition of its own, the additional Burkhardt well would only increase the transition temperature, but not change the general picture, and therefore both models should behave similarly. Clearly, the scenario proposed here must be subject to further scrutiny before accepting it. In particular, to confirm that our interpretation carries over to the $2 \mathrm{D}$ case, it would be necessary to carry out large-scale simulations (systems of size at least $1000 \times 1000$ at finite temperature) to verify whether $C(r)$ crosses over, as $r$ increases, to an asymptotic, constant value, signaling a flat phase. Such simulations are presently beyond our computing capabilities and therefore we have not been able to verify this prediction. We hope that this work encourages numerical work along this line to definitively settle this long-standing issue. Progress in this direction would influence a much broader field, namely phase transitions in disordered systems, hence the relevance and interest of further research on this problem. In this context, we conclude by stressing the importance of the results presented here. Even if they were only relevant in 1D and the scenario were not (as we believe it is) applicable to 2D super-roughening, our results constitute an example of a phase transition in a $1 \mathrm{D}$ disordered system which can be amenable to additional analytical work and lead to new insights on the exciting issue of disordered systems. 
This work has been supported by the Ministerio de Ciencia y Tecnología of Spain through grants BFM2000-0006 and BFM2003-07749-C05-01 (SA and AS). Work at Los Alamos is performed under the auspices of the US Department of Energy.

\section{REFERENCES}

[1] Plischke M. and Bergersen B., Equilibrium Statistical Physics (World Scientific, Singapore) 1994.

[2] Weeks J. D. and Gilmer G. H., Adv. Chem. Phys., 40 (1979) 157; Weeks J. D., Ordering in Strongly Fluctuating Condensed Matter Systems, edited by RISTE T. (Plenum, New York) 1980; van Beijeren H. and Nolden I., Structure and Dynamics of Surfaces, edited by Schommers W. and von Blackenhagen P. (Springer, Berlin) 1987.

[3] Toner J. and DiVincenzo D. P., Phys. Rev. B, 41 (1990) 632.

[4] Batrouni G. G. and Hwa T., Phys. Rev. Lett., 72 (1994) 4133.

[5] Shapir Y., Dynamics of Fluctuating Interfaces and Related Phenomena, edited by KIM D., PARK H. and Kahng B. (World Scientific, Singapore) 1997.

[6] Rieger H., Phys. Rev. Lett., 74 (1995) 4964.

[7] Marinari E., Monasson R. and Ruiz-Lorenzo J. J., J. Phys. A, 28 (1995) 3975.

[8] Lancaster D. J. and Ruiz-Lorenzo J. J., J. Phys. A, 28 (1995) L577.

[9] Zeng C., Middleton A. A. and Shapir Y., Phys. Rev. Lett., 77 (1996) 3204.

[10] Blasum U., Hochstättler W., Moll U. and Rieger H., J. Phys. A, 29 (1996) L459; Rieger H. and Blasum U., Phys. Rev. B, 55 (1997) R7394.

[11] Coluzzi B., Marinari E. and Ruiz-Lorenzo J. J., J. Phys. A, 30 (1997) 3771.

[12] Sánchez A., Bishop A. R., Cai D., Grønbech-Jensen N. and Domínguez-Adame F., Physica D, 107 (1997) 325.

[13] Ruiz-Lorenzo J. J., Proceedings of the VIII Spanish Meeting on Statistical Physics FISES '97, edited by Cuesta J. A. and SÁnchez A. (Editorial del Ciemat, Madrid) 1998.

[14] Rieger H., Phys. Rev. Lett., 81 (1998) 4488.

[15] Sánchez A., Bishop A. R. and Moro E., Phys. Rev. E, 62 (2000) 3219.

[16] Cuesta J. A. and SÁnchez A., J. Phys. A, 35 (2002) 2377.

[17] Ares S., Cuesta J. A., Sánchez A. and Toral R., Phys. Rev. E, 67 (2003) 046108.

[18] Burkhardt T. W., J. Phys. A, 14 (1981) L63.

[19] Chui S. T. and Weeks J. D., Phys. Rev. B, 23 (1981) 2438.

[20] Cuesta J. A. and Sánchez A., J. Stat. Phys., 115 (2004) 869; cond-mat/0306354 (2003).

[21] In addition, the quadratic surface tension term is much easier and faster to simulate than the absolute-value term.

[22] Newman M. E. J. and Barkema G. T., Monte Carlo Methods in Statistical Physics (Oxford University, Oxford) 1999.

[23] IBA Y., Int. J. Mod. Phys., 12 (2001) 623.

[24] Toral R., Proceedings of the Third Granada Lectures in Computational Physics, edited by Garrido P. L. and Marro J., Lect. Notes Phys., Vol. 448 (Springer-Verlag, Berlin-Heidelberg) 1994.

[25] Schneider T. and Stoll E., Phys. Rev. B, 22 (1980) 5317.

[26] Ares S. and SÁnchez A., unpublished (2004).

[27] Ares S., SÁnchez A. and Bishop A. R., unpublished (2004).

[28] SÁnchez A. and Bishop A. R., unpublished results (2001). 